\title{
ATEXTURE CLASSIFICATION USING RANDOM FOREST AND DECISION TREE
}

\author{
MOHAMMED MAJEED AL-SAMARAI ${ }^{1}$, MD JAN NORDIN ${ }^{2}$, GHASSAN \\ JASIM AL-ANIZY \\ ${ }^{1}$ Ministry of Electricity, Almansor, Baghdad, Iraq \\ ${ }^{1,2}$ Universiti Kebangsaan Malaysia, UKM, Bangi, Malaysia, Faculty of Information \\ Science and Technology \\ Email: almula1981@yahoo.com \\ Revised July 2015 \\ ABSTRACT. Texture analysis is considered fundamental and important in the fields \\ of pattern recognition, computer vision and image processing. Analysis of the \\ textures involves texture features extraction and selection, and plays an essential \\ role in the classification and segmentation of textural features. In this study have \\ compared two texture classification methods based on the Random Forest $(R F)$ and \\ Decision Tree (DT) classifiers by using a combination method between various \\ extraction features, such as bi-orthogonal wavelet transform, gray level histogram \\ and edge detection. Experiments were conducted on two different databases. The \\ first texture database captured digital images for testing multi-class machining \\ processes, and the second database was collected from the Brodatz album. The \\ results have revealed that $R F$ and DT have yielded higher classification precision.
}

Keywords: Supervised Random Forest, Supervised Decision Tree, Feature Extraction, Texture Classification

1. Introduction. Texture has been defined as incorporating the recurring patterns with a typical frequency whereas, texture analysis involves classifying or segmenting textural attributes based on the shape of a smaller aspect, solidity and course of persistence [1]. Furthermore, texture classifications have become a common pattern and have been employed in many fields, such as; satellite image classification, image retrieval, remote sensing images, industrial images and medical images classification [2]. The main goal of texture classification is to classify images into homogeneous classes that have the same textural pixels [3]. [4] has proposed a pattern recognition theory which runs surface roughness classification by comparing the investigated surface with other common surfaces. Also, [5] used two commonly extracted features, namely, histogram and edge detection and idea principal component analysis based on an artificial neural network technique, to classify a database of multi-class machined textures. Another study conducted by [6] used a real time visual inspection system for the enhancement of rail images based on the local normalization (LN) method. [7] have proposed a multi-scale algorithm using unsupervised extraction. They implemented the method based on the most significant edges. They designed the algorithm in order to deal with speckled SAR (Synthetic-aperture radar) images. [8] perform an edge detection method based on the Canny Edge Detection algorithm to extract the main image schematic and shape. They also used the K-means algorithm in order to differentiate among the regions in the image for improving the matching process that occurs between the user query and the needed database images' features. Also, [9] have compared and evaluated six methods to segment three sets of images that have four Brodatz textures each. The methods are the Haralick method, run length, gray level difference, Fourier spectrum, Gabor features and pixel statistics. Their results have shown that Gabor features and Haralick features were the best among others. [10] proposed an algorithm for texture classification to extract features by using Nonsubsampled Contourlet Transform and Least Square support 
Vector Machine classifiers. Recently, research works usually depend on supervised classifiers or unsupervised classifiers in texture classification approaches. For example, [11] examined the Random Forest (RF) classifier to improve the image segmentation method and measure their importance in classification. [12] used the support vector machine technique to classify five texture images based on the prospect weighted incorporation method of three feature extraction methods, including the Gray-Level Co-occurrence Matrix, Semivariogram Function and Gaussian Markov Random Fields. [13] proposed approaches that evaluated on the brodatz and CUReT databases and compared various texture classification methods. [14] have compared a number of features that were extracted from the Haralick method, Fourier transforms, Gabor filters and pixel patterns to classify Brodatz textures.

This work is mainly based on a two stage process: the image analysis stage and the image classification stage. The aim of the first stage is to apply a histogram, edge detection, and bi-orthogonal wavelet feature extraction techniques on two different types of homogenous databases. The second stage is to classify the input vector of each image using Random Forest (RF) and Decision Tree (DT) classifiers. This paper has been organized as follows: Section 2 explains our methodology; Section 3 presents the evaluation of the experimental results; and Section 4 presents the conclusion.

2. The Methodology. The methodology presented in this study is applied to the textured images to be later on classified in RF and DT classifiers. The aim is to show the tested image in a vector of features, which is referred to as the input vector which will then be directed to the RF or DT classifiers for classification. Hence, this method begins with the first stage, which is the features extraction stage using histogram, edge detection and bi-orthogonal wavelet methods from the tested image as depicted in Figure. 1. The second stage is the choice of the input vectors from the extracted features to be classified using RF and DT classifiers.

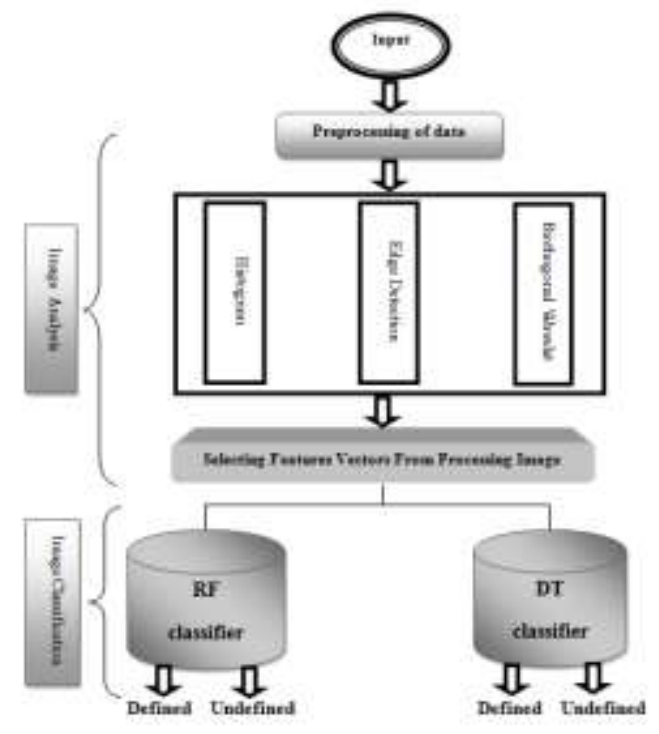

Figure.1: Proposed Model for Image Analysis and Classification via selecting the vector of the best extraction method.

2.1. Databases. The database used consists of two parts; the first part is considered the main part in our work which is the engineering machined surface specimens and, its six types of machining processes, namely, turning, grinding, horizontal milling, vertical milling, lapping, and shaping. The second part, Brodatz textures originate from a book called "Textures: A Photographic Album for Artists and Designers", and published in 1966 [15]. The pictures in this book were taken by Phil Brodatz, a highly skilled professional photographer.

2.2 .Extraction of Texture Features. The process of representing or modifying the original data is called feature extraction. Statistical, structural, and spectral strategies are basically used in image processing for detailing the texture of a region. The process of choosing an appropriate subset of features is known as feature selection. The purpose of feature selection is to enhance the consequence of learning. In the next 
subsections, we discuss the feature extraction methods and the means of choosing the input vector from every method.

2.3. Histogram Computation Method. The histogram in image processing indicates the histogram image density values. Calculating the histogram of an image provides a vector of length 256 that expresses the image. This image is then used as the input vector for the chosen classifier. The output gives 36 of these input vectors from the other surfaces that were worked on or whose databases were engineered and divided into a total of six matrices based on the type of the class. Each of these six matrices were of a uniform size $(6 \times 256)$. Along with these matrices, the output also gives us 110 vectors from the Brodatz textures databases, which are organized by a matrix of size $(110 \times 256)$. These matrices consist of columns that are used as the input vectors in order to train each data set individually and to test them for the classifiers.

2.4. Method for Detection Edge. Edge detection is a basic operation in image processing to find a change in density. The edges in any image refer to the abrupt change in the density gradient in the image. The edge detection method has been applied to obtain new images with discovered edges [16]. Based on edge the detection method, edges of machined surface images are horizontally and vertically extracted at $0^{\circ}$ and $90^{\circ}$ degrees, and a Canny edge method is applied to obtain new images with edges detected. The variance and mean of each of these images are recorded and correspondingly saved in a vector at the length column, which equals to the number of column $(1280 \times 2)$ elements. These column elements are applied to each of the images, giving 36 vectors from the data set of the machined surfaces, which are divided into six matrices, based on the type of class, with a $(2560 \times 6)$ matrix. These column elements also give 110 vectors of textures from the Brodatz database; they are organized by a $(110 \times 2560)$ matrix size. Each data set is then trained by these matrices' columns, which are separately employed as input vectors, and are then used for evaluation of the classifier.

2.5. Biorthogonal Wavelet Method. Biorthogonal wavelets are the more desirable ones in image processing applications. Image contents are prevented from shifting between sub-bands by these symmetrical wavelets allowing the image boundaries an extension. To carry out the image application the two-dimensional wavelets transform and lead to a deterioration of approximate coefficients at level i in four components as shown in Figure 2. The image also shows approximation at level $i+1$, and the details only in the orientations that exist (horizontal, diagonal and vertical).

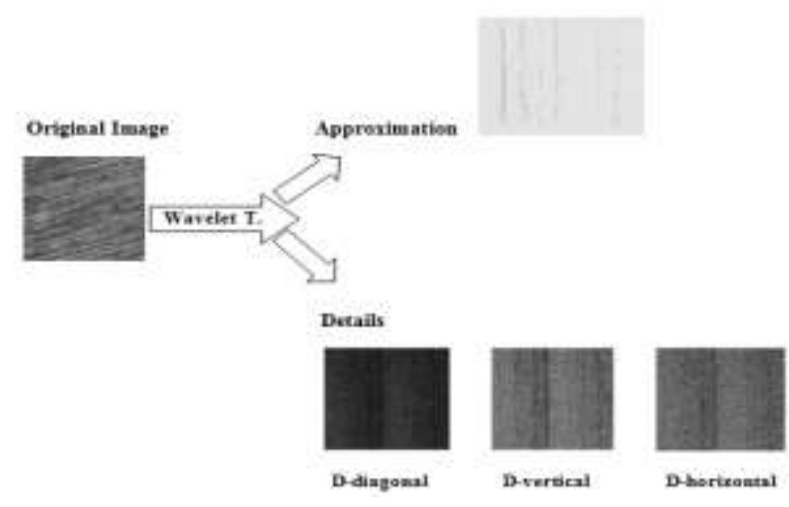

Figure 2: Two D - Wavelet Transformation

The components with an extremely higher frequency have been extracted from the images, so as to have the ideal precision in the arrangement of the two databases; which offer better performance, and consequently the two dimensional bi-orthogonal makes over the tested images; and then extract the three details (horizontal, diagonal and vertical) with $0^{0}, 90^{\circ}$ and $45^{\circ}$ being their orientation respectively. Several tests were performed on the various types of selected features. The final selection was formulated with the following:

1. Sum: total number of pixel amount that exist in a detailed component.

2. Maximum: the leading component in a varying selection in the line of a myriad of extents.

3. Mean: the total amount of components in a diverse selection. 
4. Standard deviation (std): A row vector that comprises of the standard deviations of the components of each column and can be expressed as.

$s=\left[\frac{1}{n} \sum_{i=1}^{n}\left(X_{i}-\bar{X}\right)^{2}\right]^{\frac{1}{2}}$.where $\bar{x}=\frac{1}{n} \sum_{i=1}^{n} x_{i}$

5. Median: this is the median value of components in a wide number of choices.

6. Range: this constitutes the least and most amounts of the components in line with the different extents of selections.

The aforementioned 6 features were cumulated from the 3 details formed by transformation of the image, and then its collection so as to get a final trajectory measuring as long as 18 elements demonstrating each image. Based on each data set, the production courses are separated into mediums. The columns of these matrices are sized at $(36 \times 18)$ and $(110 \times 18)$ for database 2 which are utilized for testing and training the classifiers just as how it is usually done with the other methods.

2.6. CombinationMethod. Three stages involve the attaining of this method. Firstly, the features extraction step which cover histograms, edge detection, bi-orthogonal wavelets which are then implemented to the image texture. The selection of the input vectors from the features that were extracted to make a combination between three of the vectors comes second in the line of steps and we get the vector length (2834). Thirdly these vectors classified by the RF and DT classifiers as shown in Figure. 3.

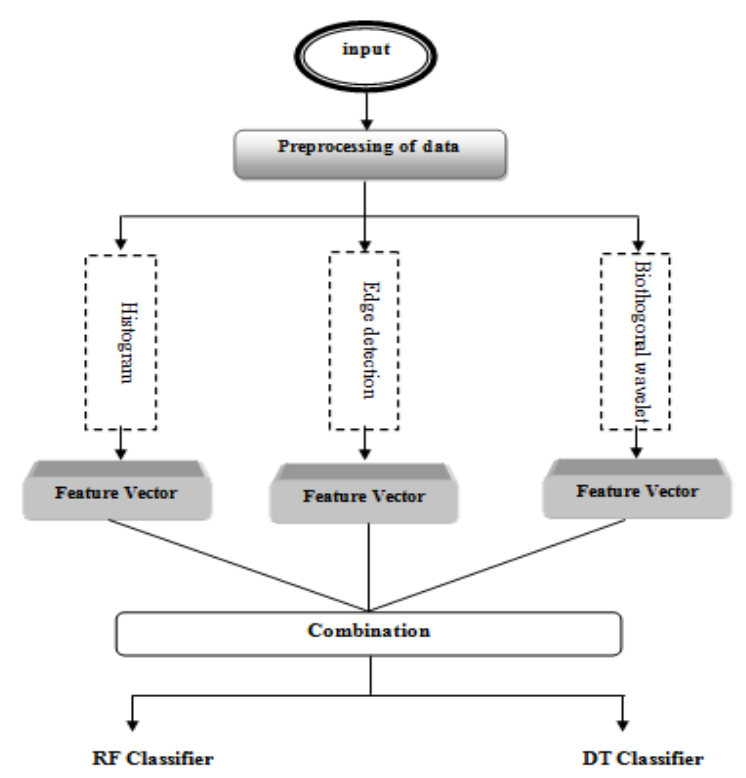

Figure. 3 Proposed Model for Image Analysis and Classification via combining the vectors of the extraction methods

2.7. RF-Based Classification. The supervised classifier used primarily has been based on Random Forest. A collection of tree structured classifiers $\{\mathrm{h}(\mathrm{x}, \Theta \mathrm{k})\}$ exist in a random forest classifier. The $\{\Theta \mathrm{k}\}$ are the identical distributed random vectors that are independent. Each tree casts a vote for the most popular class of input $\mathrm{x}$; this classifier has been built and trained using the following settings and guidelines:

The trees have been built based on the following algorithm [18]:

1. The number of training cases can be $\mathrm{N}$ and the number of variables in the classifier can be $\mathrm{M}$.

2. The number $\mathrm{M}$ of input variables is used to determine the assessment at a node of the tree; $\mathrm{m}$ should be much less than $\mathrm{M}$.

3. A training set is selected or the tree by choosing $\mathrm{n}$ times replacing it from all $\mathrm{N}$ number of available training cases (i.e., by taking a bootstrap sample). The rest of the cases can be used to estimate the errors by 
predicting their classes.

4. A random selection of $m$ variables is done for each node of the tree. The best split based on the $m$ variable is calculated.

5. The trees are fully grown and not pruned.

2.8. DT-Based Classification. The purpose of the second case is to learn the properties of applications of the supervised DT classifier on data sets that are not similar and tested by utilizing multiple removal methods described previously. These removal methods are the same methods that were used in the first study case section, such as histogram, edge detection, bi-orthogonal wavelet transform. A classifier is shown as an algorithmic separation of the instance space. A rooted tree is formed by the nodes existing in the decision tree, which means it is an engaged tree consisting of nodes called "root" and one which does not harbor inbound limits. The existing nodes consist of a single inbound edge each. An outbound edge existent node can be considered the inner node. The rest of the nodes are referred to as final nodes. Each inner node divides the case area to more than one sub-area in a decision tree, according to specific distinct numbers of the contributing traits. The large number of cases has trials that take into account each trait in such a manner so as to have the circumstance area divided based on the importance of the trait. The classification is carried out by directing the instances from the root to the leaf of the tree based on the outcome of the tests along the way.

3. Experimental Result. This section presents the overall outcome of our work and rates of precision depending on the RF and DT classifiers. The analysis and discussions of these results have been illustrated in detail in order to carry out a wide comparison and view about the best texture analysis methods, and also the best classification techniques. The first database used in this study comprised 36 multi-class engineering surface images of size $(1024 \times 1280)$. Furthermore, the second database has been prepared by collecting the images $(1$ to 110$)$ of size $(640 \times 640)$ from the Brodatz album.

3.1. Results of RF-Based Texture Classification. The test outcomes of three distinct textural features extraction techniques based on the machined textures database have revealed that, the employment of histogram, edge detection, and bi-orthogonal wavelet transform and combination methods have yielded the higher classification precision. Figure. 4 shows the variations in classification rates. Based on this figure, we can note that, using the feature extraction method and the combination method with RF is the best composition for classification, since the RF yields a higher precision rate in classification that means less complexity and less time in training. This is proved by using 36 multi-class engineering surface images.

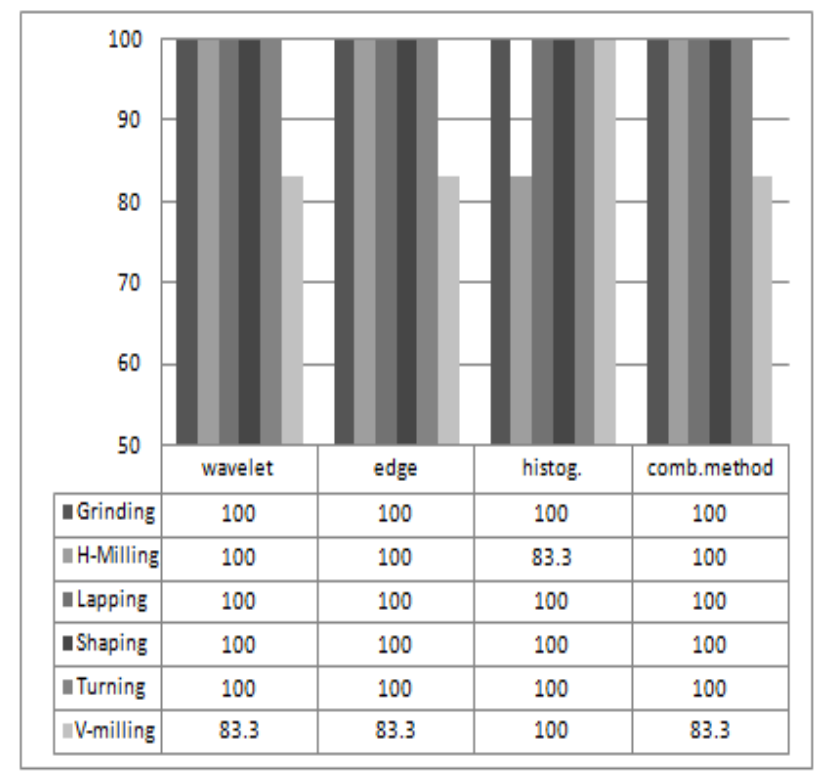

Fig 4 Precision using six different class databases based on RF 
The training and recalling have been performed to all images, using the same supervised RF model. As mentioned above, in this work, the Brodatz texture images have been used as a second database for verifying and confirming the precision of the results when using the engineering machined surfaces database. Table 1 reveals that the precision of results of the classified Brodatz textures ranges between $98.36 \%$ and $99.09 \%$ respectively, and it nearly matches the results obtained by our first data.

TABLE 1

PRECISION USING BRODATZ TUXTURES DATABASE
\begin{tabular}{|c|c|c|c|}
\hline $\begin{array}{c}\text { Analysis } \\
\text { Method }\end{array}$ & Wavelet & Edge & Histogram \\
\hline $\begin{array}{c}\text { Defined } \\
\text { Samples }\end{array}$ & 109 & 106 & 109 \\
\hline $\begin{array}{c}\text { Undefined } \\
\text { Samples }\end{array}$ & 1 & 3 & 1 \\
\hline Precision & $99.09 \%$ & $98.36 \%$ & $99.09 \%$ \\
\hline
\end{tabular}

3.2. Results of DT-Based Texture Classification. The DT performance for the three various features extraction methods using the machined database (Grinding, H-Milling, Lapping, Shaping, Turning, V-Milling) are shown in Figure 5. Based on this figure, we cannot that the experimental results show that using the bi-orthogonal wavelet transformation method and combination method for features extraction yields the highest precision rates in classification.

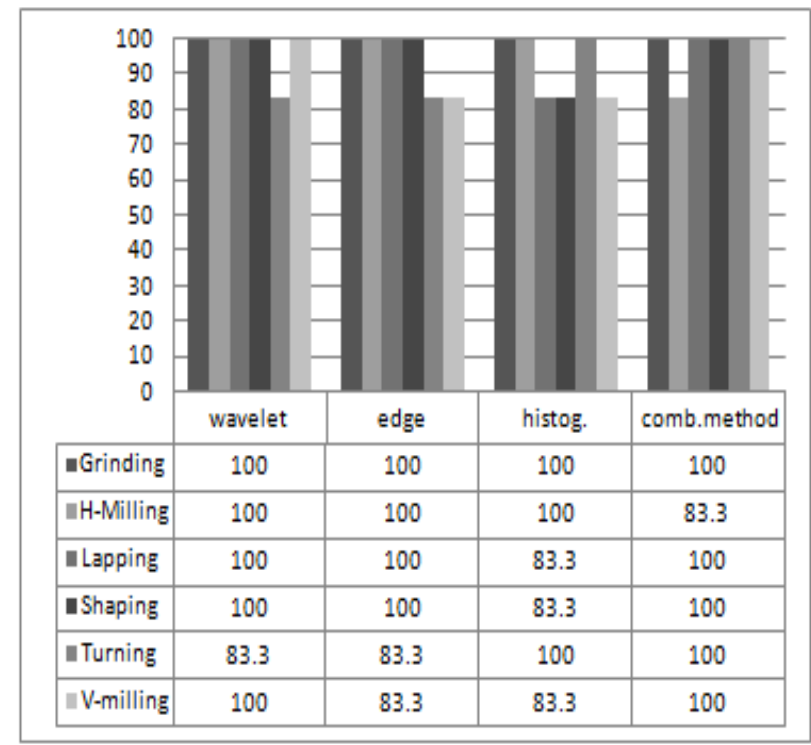

Fig 5 Precision using six different class databases based on DT

The training and recalling have been performed to all images using the same supervised DT model. As mentioned earlier in this work, the Brodatz texture images have been used as a second database for verifying and confirming the precision of the results when using the engineering machined surfaces database. Table 2 reveals that the precision of the results of the classified Brodatz textures ranges between $97.53 \%$ and $99.74 \%$ respectively, and it is higher than the results obtained by our first database. 
TABLE 2

PRECISION USING BRODATZ TUXTURES DATABASE

\begin{tabular}{|c|c|c|c|}
\hline $\begin{array}{c}\text { Analysis } \\
\text { Method }\end{array}$ & Wavelet & Edge & Histogram \\
\hline $\begin{array}{c}\text { Defined } \\
\text { Samples }\end{array}$ & 109 & 109 & 109 \\
\hline $\begin{array}{c}\text { Undefined } \\
\text { Samples }\end{array}$ & 1 & 1 & 1 \\
\hline Precision & $99.74 \%$ & $97.53 \%$ & $98.42 \%$ \\
\hline
\end{tabular}

3.3.Compared RF Results with DT Results. The proposed method has observed the effect of using two supervised classifiers on two different types of textures databases based on three various features extraction methods previously mentioned. It is noticeable that the classification done to the second database confirms the classification results of the machined textures, due to the comparison of the results in Figure 6 with the results in Figure 7. The comparison of the RF and DT classifiers regarding the precision rate and complexity have facilitated to classify and identify the type of machine process for the tested image, in terms of the engineering machined surfaces database.

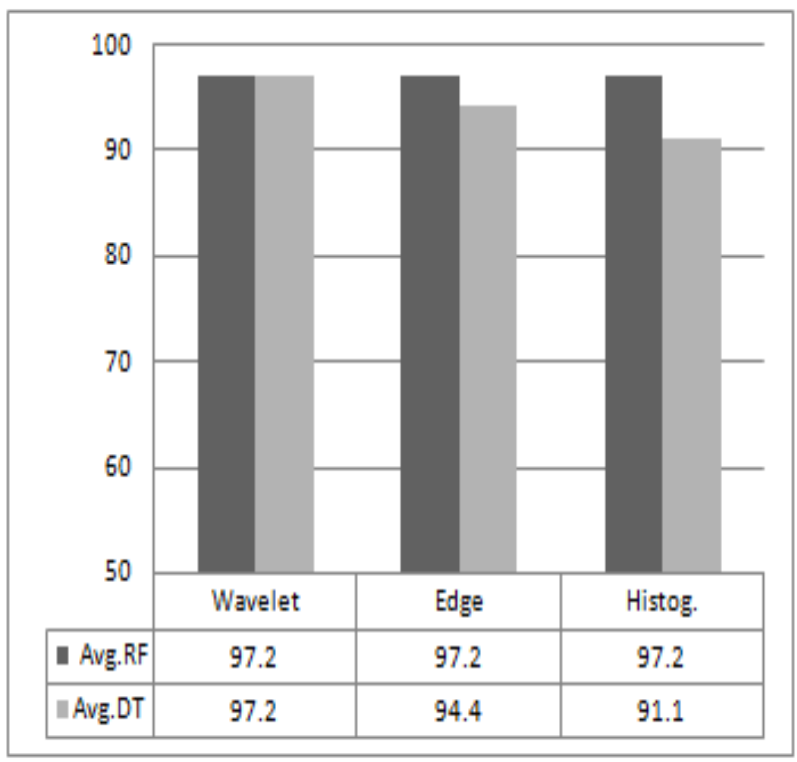

Fig. 6. Precision comparison for the three features extraction methods based on the first database using RF and DT classifiers. 


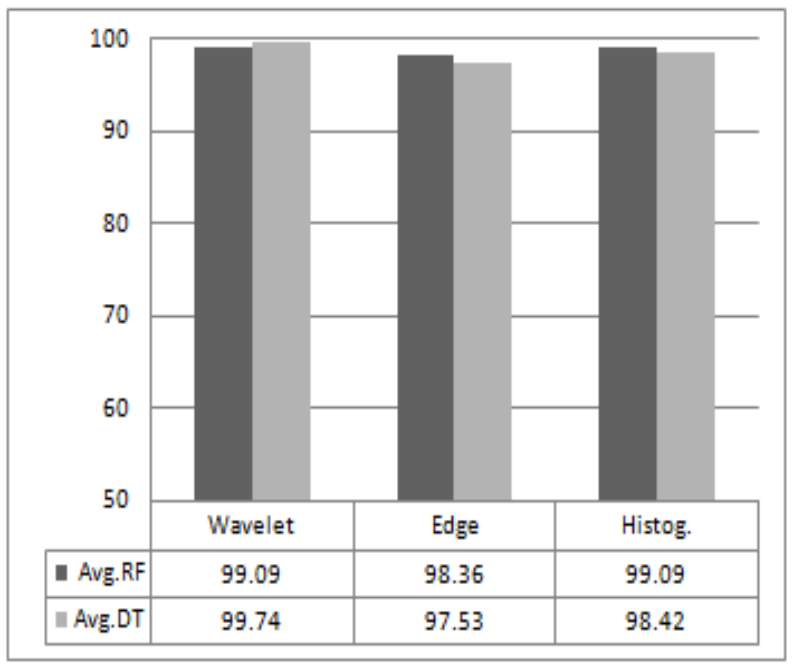

Fig. 7. Precision comparison for the three features extraction methods based on the second database using RF and DT classifiers.

4. Conclusion. The overall aim of this study is to investigate the possibilities of the texture classification process, which mainly depends on efficient image analysis by features extraction.

The results of the analysis had revealed that the RF classifier gives a higher precision rate in classifying the texture images and much less time in training. The RF classifier achieved $97.2 \%$ precision rate and used three features extraction and combination methods in classification of the machined work-pieces. It achieved a precision between $98.36 \%$ and $99.09 \%$ in classification of the Brodatz textures. The precision rate of DT ranges between $91.1 \%$ and $97.4 \%$ and the DT classifier achieved a precision rate of $97.4 \%$ when used with the combination method in classifying the machined database, while the precision rate ranged between 99.42\% and $99.74 \%$ for the Brodatz textures. Both, RF and DT classifiers have been proven as successful for studying classification in image processing. The above results have been compared with the work of [19], in which they have used the Artificial Neural Network, which yielded an accuracy of $95.4 \%$ for the machined work-pieces dataset and $86.5 \%$ for the Brodatz textures dataset. Hence conclude that, the current study has achieved a higher and better accuracy.

Acknowledgements. The authors would like to express their gratitude to the National University of Malaysia for supporting this research.

\section{REFERENCES}

[1] Tuceryan, M. and A.K. Jain, 1998. Texture Analysis. In: Handbook of Pattern Recognition and Computer Vision, Chen, C.H., L.F. Pau and P.S.P. Wang (Eds.). World Scientific Publishing, New Jersey, USA., pp: 207-248.

[2] Liu, H., Y. Lan, Q. Wang, R. Jin, E. Song and C.C. Hung, 2012. Fast weighted k-view-voting algorithm for image texture classification. Opt. Eng., Vol. 51, No. 2.

[3] Ren, H., Y. Lan and Y. Chen, 2012. An improved k-view-voting based texture classification method. Proceeding of the 4th IEEE International Conference on Computational and Information Sciences, August 17-19, 2012, Chongqing, pp: 522-525.

[4] Shi, W. M., S.P. Lim and K.S. Lee, 1995. Surface roughness classification using pattern recognition theory. Opt. Eng., 34: 1756-1760.

[5] Ashour, M.W., F. Khalid and M. Al-Obaydee, 2013. Supervised ANN classification for engineering machined textures based on enhanced features extraction and reduction scheme. Proceeding of the International Conference on Artificial Intelligence in Computer Science and ICT, November 25-26, 2013, Langkawi, Malaysia, pp: 71-80.

[6] Li, Q and S. Ren, 2012. A real-time visual inspection system for discrete surface defects of rail heads. IEEE Trans. Instrum. Meas., 61: 2189-2199. 
[7] Alonso, M.T., C. Lopez-Martinez, J.J. Mallorqui and P. Salembier, 2011. Edge enhancement algorithm based on the wavelet transform for automatic edge detection in SAR images. IEEE Trans. Geosci. Remote Sens., 49: 222-235.

[8] Ramamurthy, B. and K.R. Chandran, 2011. CBMIR: Shape-based image retrieval using canny edge detection and k-means clustering algorithms for medical images. Int. J. Eng. Sci. Technol., 3: 1870-1877.

[9] Manian, V. and R. Vasquez, 1997. Feature analysis for scaled and rotated texture segmentation. Comput. Ind. Eng., 33: 445-448.

[10] Liu M., Y. Hou, X. Guo, Z. Huan and D. Yang, 2009. Texture classification using nonsubsampled contourlet transform and LS-SVM. Proceedings of the 2nd International Congress on Image and Signal Processing, October 17-19, 2009, Tianjin, pp: 1-5.

[11] Mahapatra, D., 2014. Analyzing training information from random forests for improved image segmentation. IEEE Trans. Image Proc., 23: 1504-1512.

[12] Pharsook, S., T. Kasetkasem, P. Larmsrichan, S. Siddhichai, T. Chanwimaluang and T. Isshiki, 2011. The texture classification using the fusion of decisions from different texture classifiers. Proceedings of the 8th Electrical Engineering/Electronics, Computer, Telecommunications and Information Technology, May 17-19, 2011, Khon Kaen, pp: 1003-1006.

[13] Shu, L. and A.C.S. Chung, 2010. A new subspace learning method in fourier domain for texture classification. Proceeding of the 17th IEEE International Conference on Image Processing, September 26-29, 2010, Hong Kong, pp: 4589-4592.

[14] Augusteijn, M.F., L.E. Clemens and K.A. Shaw, 1995. Performance evaluation of texture measures for ground cover identification in satellite images by means of a neural network classifier. IEEE Trans. Geosci. Remote Sens., 33: 616-626.

[15] Brodatz, P., 1966. Textures: A Photographic Album for Artists and Designers. Dover Publications Inc., New York, USA., ISBN-13: 978-0486216690, Pages: 128.

[16] Reza, A.M., 1999.Spire Lab, and UWM, "Wavelet Characteristics-What Wavelet Should I Use?," White Paper.

[17] Horng, M.H., 2003. Texture feature coding method for texture classification. Opt. Eng., 42: 228-283.

[18] Breiman L., 2001. Random forests. Mach. Learn., 45: 5-32.

[19] Ashour, M. W. M. K. M. Mahar, and M. F. Hussin, 2007. "Artificial Neural Network for Texture Classification Using Several Features: A Comparative Study,” ACIT. 